Cite as:

Takousi, M., Schmeer, S., Manaras, I., Olympios, C.D., Fakiolas, C.N., Makos, G., \& Troop, N.A. (2016). Translation, adaptation and validation of the Coronary Revascularization Outcome Questionnaire into Greek. European Journal of Cardiovascular Nursing 15 (2), 134-141.

\title{
Translation, Adaptation and Validation of the Coronary Revascularization Outcome Questionnaire (CROQ) into Greek
}

\begin{abstract}
Maria G. Takousi $\mathrm{MSc}^{1}$, Stefanie Schmeer PhD, CPsychol ${ }^{1}$, Irene Manaras PhD, $\mathrm{CPsychol}^{2}$, Christoforos D. Olympios $\mathrm{MD}^{3}$, Constantine N. Fakiolas $\mathrm{MD}^{4}$, Georgios Makos MD ${ }^{5}$, Nick A. Troop PhD, CPsychol ${ }^{1}$
\end{abstract}

${ }^{1}$ Department of Psychology, Health \& Human Sciences Research Institute, University of Hertfordshire, Hatfield, UK

${ }^{2}$ Health Psychologist, Department of Psychology, IST College, Athens, Greece

${ }^{3}$ Interventional Cardiologist, Head, Department of Cardiology, Thriasio Hospital,

Elefsina, Greece

${ }^{4}$ Interventional Cardiologist, Head, Department of Interventional Cardiology, Tzaneio Hospital,

Piraeus, Greece

${ }^{5}$ Cardiothoracic Surgeon, Head, Department of Cardiothoracic surgery, Metropolitan Hospital, N. Faliro, Greece

Correspondence to: Maria Takousi: m.takousi2@herts.ac.uk tel: 00306937392080 Fax:00302109418687. 


\section{Abstract}

Background and aims: Evaluating the impact of coronary revascularization (CR) on patients' Health Related Quality of Life (HRQoL) with a patient-based and disease-specific tool is important for drawing conclusions about treatment and outcomes. This study reports on the translation, adaptation and psychometric evaluation of a Greek version of the Coronary Revascularisation Outcome Questionnaire (CROQ-Gr).

Methods: A total of $609(81.7 \%$ male $)$ patients who had undergone coronary revascularisation (PCI or $\mathrm{CABG}$ ) were recruited from four hospitals in Athens. After translating the CROQ into Greek, a preliminary qualitative study and a pilot quantitative study were conducted. A full psychometric evaluation was carried out on the main study's data.

Results: The psychometric evaluation demonstrated that the CROQ-Gr is acceptable to patients (high response rate, low missing data) and has a good level of reliability [internal consistency (>0.70), test-retest reliability $(>0.90)]$ and validity (both content and construct validity).

Conclusions: The results of this study show the CROQ-Gr to be a psychometrically rigorous patient-based measure of outcomes of coronary revascularisation. It would be appropriate for use in evaluative research as well as a routine clinical tool to aid cardiologists in monitoring the outcomes of care.

Key words: Translation, Validation, HRQoL, PCI, CABG, CROQ 


\section{Introduction}

Cardiovascular disease is one of the leading causes of mortality globally accounting for approximately 17 million deaths annually (1). With respect to medical treatment, invasive and surgical coronary procedures have been developed and are widely used for treating coronary heart disease (CHD). The two most commonly used types of coronary revascularization (CR) are Percutaneous Coronary Interventions (PCI), formerly called Percutaneous Transluminal Coronary Angioplasty (PTCA) and generally known as stent placement, and Coronary Artery Bypass Grafting (CABG), generally known as bypass surgery. According to research evidence, these two procedures have reduced the mortality and morbidity rates of patients with coronary diseases (2).

However, health is not just a biomedical issue. Beyond the survival benefit and relief of symptoms, patients' health-related quality of life (HRQoL) should be considered (3). While there is no consensus definition of HRQoL, the most commonly adopted is that of the World Health Organization which incorporates physical, psychological and social well-being (4).

The literature on quality of life demonstrates that monitoring the impact of an intervention on patients' HRQoL allows health professionals to enhance and promote patients' wellbeing (5), to draw valid conclusions about the effectiveness of a specific treatment, to aid clinical decision making about the type of treatment that best suits individual needs (6), to clarify issues requiring further research such as highlighting additional factors (e.g. duration of hospital stay after revascularisation) that may influence outcome in the short and long term and, finally, to develop specific (tailored) interventions for enhancing patients' wellbeing (7). Therefore, a great deal of research has been carried out using patient-based instruments in order to determine the level of patients' HRQoL before and after cardiac surgery (8). However, no clear conclusions can be 
drawn because the literature provides inconsistent results (9). The discrepancy in the literature can be partially explained by disagreement regarding the domains of HRQoL that patient-based instruments should measure and also by the use of generic instead of disease-specific instruments that assess patients' perception of health status.

Until recently, there were no validated disease-specific patient-based instruments for coronary revascularisation (10). Schroter and Lamping (12) therefore developed the Coronary Revascularisation Outcome Questionnaire (CROQ). The use of this disease-specific measure might provide further and more accurate knowledge regarding the impact of CR on patients' wellbeing. The CROQ (11) was developed to evaluate HRQoL and health outcomes before and after the two common operational coronary procedures, PCI and CABG, capturing aspects of recovery not addressed in other heart disease questionnaires.

The CROQ has four versions, a pre- and (3-month) post-revascularization version for each of the PCI and CABG treatments. The two pre-revascularization versions measure four domains containing 32 core evaluative items and one descriptive item that it is not taken into account in scale scores. Specifically these domains are Symptoms, Physical Functioning, Psychosocial Functioning and Cognitive Functioning. The two post-revascularization versions include the same four domains plus two additional domains, Satisfaction with Outcome and Adverse Effects as well as two items that do not contribute to subscale scores.

Research demonstrates that the CROQ is valid and reliable in the original English version (11) as well as in its Italian (10), Persian (12) and Japanese versions (13). The literature suggests that the CROQ is a promising instrument for measuring HRQOL and health outcomes as it is the only tool that includes psychosocial and cognitive functioning as well as surgery-specific items (14). In fact, the CROQ has been used to detect the effect of treatment in the short and long term 
(11) as it is more responsive than other instruments (15) and yields a greater response rate compared to other measures (16). Studies using the CROQ suggest moderate to high effect sizes $(d \geq 0.67)$ of CR on HRQoL domains with the exception of Cognitive Functioning where a small effect was found (11). Moreover, the CROQ has been used in exploring HRQoL across various subtypes of coronary revascularization. For example, Angelini et al. (18) found no differences in patients' well-being between those treated with on- or off-pump CABG surgery. Similarly, Rogers et al. (19) found no difference in patients' HRQoL between those treated via left anterolateral thoracotomy (ThoraCAB) or via a conventional median sternotomy (17). These findings are very important, especially where the Cognitive subdomain is concerned. Previous literature suggests that patients undergoing off-pump CABG score higher in the cognitive subdomain compared to patients undergoing conventional CABG (18), whereas the CROQ was able to detect accurately the lack of cognitive differences after one or other treatment, a finding that is supported by recent systematic reviews where authors have focused on studies using specialised methods of cognitive testing $(19,20)$.

Clearly the CROQ is an essential patient-based measure of disease-specific HRQoL. The aim of this study is to develop a psychometrically sound Greek version of the CROQ, the CROQ-Gr.

\section{Method}

\section{Sample and data collection}

After obtaining the approval of the relevant institutional ethics committees (at the University of Hertfordshire and of the four participating hospitals), patients were recruited from Tzaneio, Thriasio, Attiko and Metropolitan Hospitals, between April 2009 and July 2012. Patients 
expected to undergo PCI or CABG in allied hospitals during the research period were eligible to participate in the study. Exclusion criteria were patients being below 18 years of age and patients with an emergency admission.

\section{Measures}

The CROQ consists of four versions; one pre-PCI, one post-PCI, one pre-CABG and one post-CABG. The two pre-operation versions are identical for the two procedures and capture four domains containing 32 core evaluative items (symptoms [7 items], physical functioning [8 items], psychosocial functioning [14 items] and cognitive functioning [3 items]) and one descriptive item that is not taken into account in computing scale scores. The two post-operation versions consist of the same four subscales as the pre-operation version as well as three items that are not taken account of in computing scale scores and two additional domains at the postoperation versions are adverse effects (6 items) and satisfaction with outcome (6 items for the PCI version and 11 for the CABG version). Total scores are rated on 0-100 scale. Higher scores indicate higher HRQoL level.

In addition to the CROQ-Gr, the Greek version of SF-36v2 ${ }^{\mathrm{TM}}$ Health Survey (SF-36) (21-23) questionnaire was used to test for construct validity. The SF-36v2 $2^{\mathrm{TM}}$ consists of 36 questions focusing on eight areas: physical functioning (PF-10 items), role limitations due to physical health problems (RP- 4 items), bodily pain (BP- 2 items), general health perceptions (GH-5 items), vitality (VT-4 items) tapping energy levels and fatigue, social functioning (SF-2 items), role limitations due to emotional problems (RE-3 items) and mental health (MH-5 items). The average score of PF, GH, RP, BP are used to create the Physical Components Summary (PCS) 
while VT, SF, RE, MH are used to create the Mental Components Summary (MCS). Higher scores indicate higher HRQoL level. Total scores are rated on 0-100 scale. Higher scores indicate higher HRQoL level.

\section{Translation and adaptation}

An instrument that is validated and widely used in one culture, is not necessarily valid and reliable in every language and culture and its adaptation requires more than a simple literal translation. However, while at least 17 methods of adaptation can be identified (24), the majority agree upon a multi-stage procedure. It involves at least two forward translators, one or two back translators, a synthesis of findings, a review by an expert panel, pre-testing and a formal psychometric testing (24). The approach adopted here is that of the International Quality of Life Assessment (IQOLA) (25-32) since this is the approach followed for the cultural adaptation of the SF-36 which is the mostly adapted instrument of HRQoL globally.

After the forward translation, a scientific panel consisting of the principal investigators and a bilingual individual revised the preliminary version of the CROQ-Gr. In addition, 25 qualitative interviews (10 with patients who had undergone PCI and 15 with patients who had undergone CABG) were conducted to ensure content validity, clarity, appropriateness of wording, questionnaire format and instructions and feasibility (regarding respondent burden). After minor modifications, the final translation back to English was conducted by another bilingual individual. Furthermore, a pilot study was conducted with a total of 112 Greek patients who had undergone PCI to demonstrate acceptability and reliability (data not shown).

\section{Statistical analysis}


Analyses are presented as suggested by the original CROQ's developers (11) and, for comparison with the original, their terminology is used. Firstly, descriptive analysis was carried out to estimate acceptability and item variance. Reliability analysis was performed using Cronbach's $a$ to estimate internal consistency and intraclass correlation coefficients (ICC) to estimate test-retest reliability (test-retest was carried out in a subsample of 20 PCI and 20 CABG patients completing measures at baseline and 3 weeks later). Construct validity (within scale) was assessed using Principal axis factoring extraction, as per the development of the original CROQ, using the 3-month post-CR data. A four-factor solution was expected and, with Varimax rotation, was set a priori with coefficient suppression at 0.35 . Construct validity (against external criteria) was evaluated using Spearman's rho correlations between the CROQ-Gr and SF-36 for 45-52 patients for each of the procedures at baseline and at 3-months post-CR. It was expected that Physical Functioning/Symptoms scales of the CROQ-Gr would correlate more strongly with the Physical Component Summary of the SF-36 than with the Mental Component Summary and that the Psychological Functioning scale of the CROQ-Gr would correlate more strongly with the Mental Component Summary of the SF-36 than with the Physical Component Summary. No predictions were made as the association between the Cognitive Functioning scale of the CROQ and the SF-36. The scoring of the SF-36 was carried out with the use of the Health Outcomes Scoring Software 3.0. Data were analysed using SPSS version 20.0

\section{Results}

Following the translation/adaptation and pilot testing of the CROQ-Gr (described in the Methods section), 472 Greek patients undergoing coronary revascularization (307 PCI and 165 CABG) were recruited for the extended psychometric evaluation of all four versions (i.e. the pre- 
and post- versions for PCI and CABG). Of these, $76.01 \%(\mathrm{~N}=359)$ were men and mean age was $M=60.4$ years, $S D=10.8$.

\section{Acceptability and reliability (internal consistency and test-retest)}

Most of the CROQ-Gr versions have good acceptability with little missing data. Both Cronbach's $a$ and intraclass correlation coefficients (ICC) exceeded the criterion of 0.70 for all scales, demonstrating excellent internal consistency and test-retest reliability (Table 1).

\section{Table 1 about here}

\section{Content validity}

Content validity was evaluated during the development of the CROQ-Gr versions and in the preliminary translation/adaptation and pilot studies described above (which included a panel of experts as well as face-to-face interviews with patients).

\section{Construct validity (within scale)}

In addition to the high levels of internal consistency and intraclass correlation coefficients described above, support for the construct validity of the CROQ-Gr can be demonstrated from the findings of the factor analysis. For all versions, the Kaiser-Meyer-Olkin test verified sampling adequacy $(\mathrm{KMO}>.78)$ and Bartlett's test confirmed sphericity $(p<.001)$. Four factors had Eigen values greater than 1 and items loaded on their expected factor (Table 2). The four factor model of rotated squared loadings explained between $41.7 \%$ and $50.3 \%$ of the variance, depending on the version. Results were almost identical to the original English version. 
Table 2 about here

\section{Construct validity (against external criteria)}

Construct validity against external criteria was examined through the correlations between the CROQ-Gr and the SF-36 subscales (see Table 3). As expected, the Symptoms and Physical Functioning subscales of the CROQ-Gr correlated more strongly with the Physical Component Summary of the SF-36 than with the Mental Component Summary. Conversely, the Psychological Functioning subscale of the CROQ-Gr correlated more strongly with the Mental Component Summary of the SF-36 than with the Physical Component Summary. These results were true for both procedures and for both the pre-and post-procedure versions, thus demonstrating both convergent and divergent validity. The Cognitive Functioning subscale of the CROQ-Gr was not reliably related to any subscales of the SF-36.

$\underline{\text { Table } 3 \text { about here }}$

\section{Discussion}

Following forward and back translation, interviews with patients, pilot testing and, following modifications, a full psychometric evaluation of the CROQ, the Greek version of the CROQ (CROQ-Gr) was shown to be a practical and psychometrically sound measure of health related quality of life and other health issues before and after heart revascularization. It is acceptable to patients and can be used in relation to both PCI and CABG. These findings are in accordance 
with findings reported by the English (11), Italian (10), Persian (12), and Japanese (13) versions of the CROQ.

Due to the current lack of a Greek disease-specific measure of HRQoL and health outcomes after CR, it could be argued that the development of the CROQ-Gr is essential. It is a subjective measure that enables assessment of both therapeutic procedures from the patient's point of view that, in turn, could allow greater insight concerning patients' treatment and greater confidence in decision making about the type of treatment that best meets patients' needs. Reviewers (33) suggest that, in order to draw clear conclusions about the effectiveness of CR on patients' HRQoL, shape decision making and develop appropriate policy, a combination of both types of measures (generic and specific) should be used. The non-significant correlations between some of the subscales in the CROQ-Gr and SF-36 suggests that there may indeed be aspects of quality of life that are captured in each measure that are not captured by the other; to determine the complete picture may, therefore, require the application of both.

Furthermore, when interpreting the findings, cultural issues (34) should be considered, because they could influence the total scores observed for each subscale which, in turn, could influence the conclusion about research findings. Specifically, scores obtained by the CROQ-Gr in the present study indicate that Greek patients report greater levels of HRQoL compared to English patients (11). This may underline the presence of differences in patients' perceptions of wellbeing and thus scores may be culturally influenced. It may be the case that Greek patients perceive their HRQoL as "good" despite their health issues. The literature indicates that patients in different cultures tend to selectively express symptoms as well as physical and/or psychological functioning in culturally acceptable ways (28). Their reporting is based on the concept of how people perceive and articulate their physical and mental problems. For instance, 
individuals in one culture might perceive it to be more acceptable to suppress upsetting thoughts, while in other cultures individuals might be more likely to adopt a more active way of expressing and facing problems rather than ignoring them. In fact, many researchers examining cultural adaptations of measures share the same concern (28). Thus, when researchers and clinicians interpret the literature they should bear in mind cultural issues as well.

Differences in attitudes towards lifestyle factors could also be implicated. Research findings reveal that the vast majority of Greeks report low physical activity and tend to avoid exercising, walking or running, preferring a sedentary life, driving and using elevators, especially those living in urban areas (35). Certainly this is consistent with the reports of some participants in the present study for whom items relating to walking $1 \mathrm{~km}$ or using the stairs were not considered relevant and who therefore had difficulty completing this part of the CROQ-Gr. However, this is not a static phenomenon and health behaviours such as physical activity are increasing, perhaps as a result of public educational interventions for the "therapeutic" role of physical activity that took place in Greece after its entry into the EU, particularly amongst younger women (36) and people with higher income (37).

The main limitations of the present study are the non-random sample which diminishes claims for generalizability, the likelihood of social desirability bias due to the form of administration and the lack of clinical validation.

Future research could use the CROQ-Gr to address a number of issues. Firstly, repeated assessment over time is important to track the trajectory of change within different domains. Secondly, identifying aspects of quality of life that are less likely to change following CR is important in order to plan additional support. Thirdly, the development of a shorter version would be useful for reducing respondent burden and increasing data quality, especially when 
research aims to explore many related issues (e.g. personality, mood) and thus may use many instruments.

Moreover, health professionals should be encouraged to use the CROQ-Gr for gaining a better understanding of the patient's experience which may facilitate not only doctor-patient communication and treatment decisions, but also drawing a clear conclusion about disease burden and effectiveness of rehabilitation. 


\section{References}

1. Mensah GA, Forouzanfar MH, Naghavi M, Lozano R, Ezzati M, Moran A, et al. Comparable estimates of mortality and trends for cardiovascular diseases including congenital heart disease in 21 world regions in 1990 and 2010: The global burden of diseases, injuries and risk factors study. Journal of the American College of Cardiology. 2013;61(10_S).

2. Jeremias A, Kaul S, Rosengart TK, Gruberg L, Brown DL. The impact of revascularization on mortality in patients with nonacute coronary artery disease. Am J Med. 2009 Feb;122(2):152-61.

3. Crosby RD, Kolotkin RL, Williams GR. Defining clinically meaningful change in health-related quality of life. J Clin Epidemiol. 2003 May;56(5):395-407.

4. Patrick DL, Burke LB, Powers JH, Scott JA, Rock EP, Dawisha S, et al. Patient-reported outcomes to support medical product labeling claims: FDA perspective. Value Health. 2007 Nov-Dec;10 Suppl 2:S125-37.

5. Brannon L, Feist J. Health psychology : an introduction to behavior and health. 5th ed. Belmont, Calif.: Thomson/Wadsworth; 2004.

6. Revicki DA, Osoba D, Fairclough D, Barofsky I, Berzon R, Leidy NK, et al. Recommendations on health-related quality of life research to support labeling and promotional claims in the United States. Qual Life Res. 2000;9(8):887-900.

7. Bowling A, editor. Measuring disease New York: Open University Press Inc.; 2001.

8. Sawatzky JA, Naimark BJ. The coronary artery bypass graft surgery trajectory: Gender differences revisited. Eur J Cardiovasc Nurs. 2009 Oct;8(4):302-8.

9. Koch CG, Khandwala F, Blackstone EH. Health-related quality of life after cardiac surgery. Semin Cardiothorac Vasc Anesth. 2008 Sep;12(3):203-17.

10. Pintor P. P. S, S., Colangelo, S., Dilaghi, B., Arpinelli, F., Fonzo, D., Torta, R., Sheiban, I. \& Bobbio, M. . Assessment of acceptability and comprehension of the Italian version of the coronary revascularisation outcome questionnaire (CROQ). . Hippokratia. 2002;6 (1):27-30.

11. Schroter S, Lamping DL. Coronary revascularisation outcome questionnaire (CROQ): development and validation of a new, patient based measure of outcome in coronary bypass surgery and angioplasty. Heart. 2004 Dec;90(12):1460-6.

12. Shahali S, Shaterzadeh-Yazdi, M., Goharpey, S. \& Rahim, F. Reliability of the Persian version of coronary revascularization outcome questionnaire (CROQ) in cardiac patients undergoing CABG and PTCA procedures. Journal of Clinical \& Diagnostic Research [serial online], [serial on the Internet]. 2008;

2.

13. Seki S, Kato N, Ito N, Kinugawa K, Ono M, Motomura N, et al. Translation and validation study of the Japanese versions of the Coronary Revascularisation Outcome Questionnaire (CROQ-J). Eur J Cardiovasc Nurs. 2010 May 9.

14. Baig K, Harling L, Papanikitas J, Attaran S, Ashrafian H, Casula R, et al. Does coronary artery bypass grafting improve quality of life in elderly patients? Interact Cardiovasc Thorac Surg. 2013 Sep;17(3):542-53.

15. Schroter S, Lamping DL. Responsiveness of the coronary revascularisation outcome questionnaire compared with the SF-36 and Seattle Angina Questionnaire. Qual Life Res. 2006 Aug;15(6):1069-78.

16. Ascione R, Reeves BC, Taylor FC, Seehra HK, Angelini GD. Beating heart against cardioplegic arrest studies (BHACAS 1 and 2): quality of life at mid-term follow-up in two randomised controlled trials. Eur Heart J. 2004 May;25(9):765-70. 
17. Rogers CA, Pike K, Angelini GD, Reeves BC, Glauber M, Ferrarini M, et al. An open randomized controlled trial of median sternotomy versus anterolateral left thoracotomy on morbidity and health care resource use in patients having off-pump coronary artery bypass surgery: The Sternotomy Versus Thoracotomy (STET) trial. J Thorac Cardiovasc Surg. 2012 Aug 31.

18. Alston RP. Pumphead--or not! Does avoiding cardiopulmonary bypass for coronary artery bypass surgery result in less brain damage? Br J Anaesth. 2005 Jun;94(6):699-701.

19. Kennedy ED, Choy KCC, Alston RP, Chen S, Farhan-Alanie MMH, Anderson J, et al. Cognitive Outcome After On- and Off-Pump Coronary Artery Bypass Grafting Surgery: A Systematic Review and Meta-Analysis. Journal of Cardiothoracic and Vascular Anesthesia. 2013;27(2):253-65.

20. Sun JH, Wu XY, Wang WJ, Jin LL. Cognitive dysfunction after off-pump versus on-pump coronary artery bypass surgery: a meta-analysis. J Int Med Res. 2012;40(3):852-8.

21. Ware JE. User's manual for the SF-36v2 health survey. 2nd ed. London: Quality Metric; 2007.

22. Ware JE. SF-36 health survey update. Spine 2000;25(24):3130-9.

23. Anagnostopoulos F, Niakas D, Pappa E. Construct validation of the Greek SF-36 Health Survey. Qual Life Res. 2005 Oct;14(8):1959-65.

24. Acquadro C, Conway K, Hareendran A, Aaronson N. Literature review of methods to translate health-related quality of life questionnaires for use in multinational clinical trials. Value Health. 2008 May-Jun;11(3):509-21.

25. Bullinger M, Alonso J, Apolone G, Leplege A, Sullivan M, Wood-Dauphinee $S$, et al. Translating health status questionnaires and evaluating their quality: the IQOLA Project approach. International Quality of Life Assessment. J Clin Epidemiol. 1998 Nov;51(11):913-23.

26. Gandek B, Ware JE, Jr., Aaronson NK, Alonso J, Apolone G, Bjorner J, et al. Tests of data quality, scaling assumptions, and reliability of the SF-36 in eleven countries: results from the IQOLA Project. International Quality of Life Assessment. J Clin Epidemiol. 1998 Nov;51(11):1149-58.

27. Leung YY, Ho KW, Zhu TY, Tam LS, Kun EW, Li EK. Testing scaling assumptions, reliability and validity of medical outcomes study short-form 36 health survey in psoriatic arthritis. Rheumatology (Oxford). 2010 Aug;49(8):1495-501.

28. Li L, Wang HM, Shen Y. Chinese SF-36 Health Survey: translation, cultural adaptation, validation, and normalisation. J Epidemiol Community Health. 2003 Apr;57(4):259-63.

29. Montazeri A, Goshtasebi A, Vahdaninia M, Gandek B. The Short Form Health Survey (SF-36): translation and validation study of the Iranian version. Qual Life Res. 2005 Apr;14(3):875-82.

30. Montazeri A, Vahdaninia M, Mousavi SJ, Omidvari S. The Iranian version of 12 -item Short Form Health Survey (SF-12): factor structure, internal consistency and construct validity. BMC Public Health. 2009;9:341.

31. Scientific Advisory Committee of the Medical Outcomes Trust. Assessing health status and quality-of-life instruments: Attributes and review criteria. Quality of Life Research. 2002 2002/05/01;11(3):193-205.

32. Wagner AK, Gandek B, Aaronson NK, Acquadro C, Alonso J, Apolone G, et al. Cross-cultural comparisons of the content of SF-36 translations across 10 countries: results from the IQOLA Project. International Quality of Life Assessment. J Clin Epidemiol. 1998 Nov;51(11):925-32.

33. Chen TH, Li L, Kochen MM. A systematic review: how to choose appropriate health-related quality of life (HRQOL) measures in routine general practice? J Zhejiang Univ Sci B. 2005 Sep;6(9):936-40.

34. Marquis P, Keininger, D., Acquadro, C. \& de la Loge, C. Translating and evaluating questionnaires: cultural issues for international research. In: Hays PFR, editor. Assessing quality of life in clinical trials 2nd ed. NY: Oxford University Press Inc.; 2005. p. 78-93. 
35. Milias GA, Panagiotakos DB, Pitsavos C, Xenaki D, Panagopoulos G, Stefanadis C. Prevalence of self-reported hypercholesterolaemia and its relation to dietary habits, in Greek adults; a national nutrition \& health survey. Lipids Health Dis. 2006;5:5.

36. Athyros VG, Bouloukos VI, Pehlivanidis AN, Papageorgiou AA, Dionysopoulou SG, Symeonidis AN, et al. The prevalence of the metabolic syndrome in Greece: the MetS-Greece Multicentre Study. Diabetes Obes Metab. 2005 Jul;7(4):397-405.

37. Tzormpatzakis N, Sleap M. Participation in physical activity and exercise in Greece: a systematic literature review. Int J Public Health. 2007;52(6):360-71. 
Table 1. Acceptability and Reliability of CROQ CABG and PCI 3mth post versions

\begin{tabular}{|c|c|c|c|c|c|}
\hline & & Acceptability & & Reliability & \\
\hline CROQ scale & $\begin{array}{l}\text { Score (range } \\
0-100) \text { mean } \\
\text { (SD) }\end{array}$ & $\begin{array}{l}\text { Missing data } \\
\text { (range) }\end{array}$ & $\begin{array}{c}\text { Floor / ceiling } \\
\text { effects } \dagger\end{array}$ & $\begin{array}{c}\text { Internal } \\
\text { consistency } \\
\text { (Cronbach's } a \text { ) }\end{array}$ & $\begin{array}{c}\text { Test-retest } \\
\text { (ICC) } \ddagger\end{array}$ \\
\hline \multicolumn{6}{|l|}{ CROQ-GABG_post $(n=134)$} \\
\hline Symptoms (7 items) & $93.70(7.95)$ & $0 \%(0-0.7 \%)$ & $0 \% / 39.8 \%$ & .83 & .82 \\
\hline Physical functioning ( 8 items) & $88.68(14.89)$ & $6.4 \%(0-24 \%)$ & $1.3 \% / 47 \%$ & .87 & .97 \\
\hline Psychological functioning (14 items) & $85.15(12.92)$ & $1.9 \%(0-5.8 \%)$ & $0 \% / 6.3 \%$ & .93 & .95 \\
\hline Cognitive functioning( 3 items) & $83.93(17.27)$ & $1.3 \%(0-2.6 \%)$ & $0 \% / 7 \%$ & .92 & .98 \\
\hline Adverse effects (11 items) & $84.24(12.72)$ & $0.4 \%(0-2.6 \%)$ & $0 \% / 9.2 \%$ & .84 & .87 \\
\hline Adverse effect chest ( 5 items) & $76.67(18.68)$ & $0.4 \%(0-2.6 \%)$ & $0 \% / 10.7 \%$ & .86 & .85 \\
\hline Adverse effect leg/arm (6 items) & $90.35(13.71)$ & $0.2 \%(0-2.2 \%)$ & $0 \% / 40 \%$ & .87 & .88 \\
\hline Satisfaction (11 items) & $72.55(22.98)$ & $1.8 \% 90-7.9 \%)$ & $0 \% / 5.5 \%$ & .85 & .94 \\
\hline \multicolumn{6}{|l|}{ CROQ-PCI_post $(\mathrm{n}=254)$} \\
\hline Symptoms (7 items) & $87.73(13.65)$ & $0 \%(0-0.2 \%)$ & $0 \% / 35.3 \%$ & .75 & .80 \\
\hline Physical functioning ( 8 items) & $88.55(16.15)$ & $5.4 \%(0-18 \%)$ & $1.3 \% / 48.2 \%$ & .83 & .87 \\
\hline Psychological functioning (14 items) & $84.39(15.13)$ & $1.9 \%(0-3.6 \%)$ & $0 \% / 6.3 \%$ & .84 & .86 \\
\hline Cognitive functioning ( 3 items) & $89.60(15.09)$ & $1.1 \%(0-2.4 \%)$ & $0.4 \% / 51.8 \%$ & .86 & .96 \\
\hline Adverse effects ( 6 items) & $97.32(6.12)$ & $0.6 \%(0.4-1.2 \%)$ & $0 \% / 77.3 \%$ & .81 & .89 \\
\hline Satisfaction (6 items) & $85.88(14.76)$ & $1.6 \%(0.4-5.1 \%)$ & $2.8 \% / 27.5 \%$ & .77 & .94 \\
\hline
\end{tabular}


Table 2. PFA for CROQ-Gr: 3 months post revascularization core items

Factors for PCI

1

4

Factor 1: symptoms

post chest pain
post discomfort
post shortness of breath
post angina radiates
post palpitations
post intake frequency
post troubles
Factor 2 : physical functioning
post moderate activities
post lifting
post climbing more than one
post climbing one
post bending
post walking a klm
post walking $100 \mathrm{~m}$
post bathing
Factor 3 : psychosocial functioning
post overprotective environment
post burden on others
post restriction of social activities
post go far from home
post worried about heart condition
post worried about overdoing
post worried about sudden attack
post unghtened by pain
post depressed
post frustrated / impatient
post enjoyment of life
postive outlook of health
portion

Factors for CABG

2

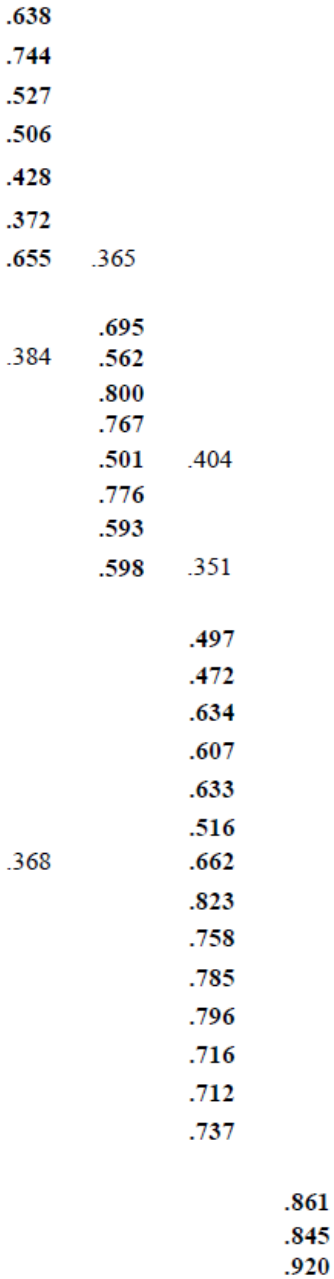


Table 3. Spearman rho correlation between CROQ and SF-36

\section{$\mathrm{PCI}$ procedure CABG procedure}

CROQ -scales

$\begin{array}{lr} & \text { SF }-36 \\ \text { PCS } & \text { MCS }\end{array}$

Baseline $(\mathrm{N}=50 / 52) \dagger$

Symptoms

$.638^{*+}$

$.719^{* *}$

$.356^{*}$

.178

Cognitive functioning

$\begin{array}{lr} & \text { SF-36 } \\ \text { PCS } & \text { MCS }\end{array}$

$.400^{*+1}$

$.291^{*}$

$.909^{*} \quad .154$

$.054 \quad .806^{* *}$

$.232 \quad .537^{* *}$

At 3months after heart surgery $(N=45 / 50) \dagger$

$\begin{array}{lllll}\text { Symptoms } & . \mathbf{4 1 7 ^ { * * }} & .172 & .098 & -.142 \\ \text { Physical functioning } & . \mathbf{6 6 7 ^ { * * }} & .225 & . \mathbf{4 9 4 ^ { * * }} & .368^{* *} \\ \text { Psychological functioning } & .369^{*} & . \mathbf{7 7 0 ^ { * * }} & .327^{*} & . \mathbf{5 2 4 ^ { * * }} \\ \text { Cognitive functioning } & -.056 & .302 & .284^{*} & -.104 \\ \text { Adverse effects (chest) } & .222 & .063 & .040 & .044 \\ \text { Adverse effects (leg/arm) } & -.006 & -.005 & -.028 & -.174 \\ \text { Satisfaction } & . \mathbf{4 1 7 ^ { * * }} & .172 & .167 & .119\end{array}$

$\dagger \mathrm{N}=\mathrm{PCI} / \mathrm{CABG}$ version ${ }^{*} \mathrm{p}<0.05 \quad * * \mathrm{p} \leq 0.01$ 